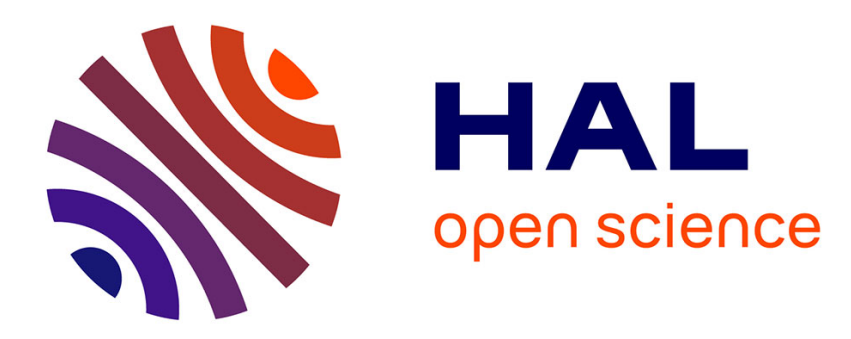

\title{
Caractéristiques des sources à plasmas nécessaires aux processus chimiques
}

\author{
J. Amouroux
}

\section{To cite this version:}

J. Amouroux. Caractéristiques des sources à plasmas nécessaires aux processus chimiques. Revue de Physique Appliquée, 1977, 12 (8), pp.1149-1161. 10.1051/rphysap:019770012080114900 . jpa00244293

\section{HAL Id: jpa-00244293 https://hal.science/jpa-00244293}

Submitted on 1 Jan 1977

HAL is a multi-disciplinary open access archive for the deposit and dissemination of scientific research documents, whether they are published or not. The documents may come from teaching and research institutions in France or abroad, or from public or private research centers.
L'archive ouverte pluridisciplinaire HAL, est destinée au dépôt et à la diffusion de documents scientifiques de niveau recherche, publiés ou non, émanant des établissements d'enseignement et de recherche français ou étrangers, des laboratoires publics ou privés. 
Classification

Physics Abstracts

\title{
CARACTÉRISTIQUES DES SOURCES A PLASMAS NÉCESSAIRES AUX PROCESSUS CHIMIQUES (*)
}

\author{
J. AMOUROUX \\ Laboratoire de Génie Chimique, E. N. S. C. P., \\ 11, rue Pierre-et-Marie-Curie, 75231 Paris Cedex 05, France
}

(Reçu le 30 décembre 1976, révisé le 20 avril 1977, accepté le 5 mai 1977)

Résumé. - Les travaux sur les sources à plasma ont permis d'établir une distinction technologique et théorique entre source à effet thermique (arc, torche) et source à excitation sélective (laser, décharge couronne...). Toutefois leurs applications au domaine de la production posent simultanément le problème :

1) du choix de la source,

2) du choix du réacteur de synthèse et de trempe.

Pour apporter des éléments de réponse à ces questions il est d'abord nécessaire d'avoir une bonne connaissance des caractéristiques physiques de la décharge (nature des espèces excitées, niveau d'excitation, durée de vie...) et de ses caractéristiques techniques de fonctionnement (technologie, limites d'emploi, puissance, rendement énergétique...). En effet la source est un des éléments de l'atelier de fabrication et sa connaissance précisera les caractéristiques de fonctionnement du réacteur haute énergie, lieu de synthèse ou d'élaboration d'espèces chimiques nouvelles (atomes, radicaux ou molécules).

Enfin le réacteur de trempe permet la recombinaison ou la conservation des espèces élaborées dans la zone haute énergie. Ainsi l'association source à plasma, réacteur haute énergie, réacteur de trempe permet la réalisation d'un procédé adapté à un objectif précis, c'est-à-dire autorisant un taux de conversion et un rendement énergétique aussi élevé que possible.

Abstract. - Plasma source studies have permitted classification of electrical discharges into two main types according to their effect : thermal (arc, torch); or selective excitation (laser, corona and glow discharges).

The application of the sources to chemical engineering introduces two questions :

1) the choice of the discharge,

2) the choice of the chemical quenching and synthesis reactor.

To answer these questions one must have a good knowledge of the physical features of the discharge (nature of the species) and of its technical characteristics (upper limits of working condition, energy yield technology).

The plasma unit is only one part in the chemical process, but its characteristics determine the parameters of the high temperature reactor where new chemical species are synthesized, whereas the quenching reactor permits the recombination of chemical species from radicals and atoms. The entire system has to be optimized in order to increase as much as possible the yield without reducing the energy efficiency.

Introduction. - La chimie des plasmas a traité au cours des 10 dernières années un nombre considérable de problèmes par l'emploi de techniques plasmas, aussi sans faire un recensement exhaustif de tous les essais entrepris, il est néanmoins possible de citer quelques exemples précis d'application :

Synthèse de produits à formation endothermique : acétylène, acide cyanhydrique, cyanogène, oxynitrures de métaux $[1,2,20,23,14]$;

(*) Communication présentée au Congrès National de Physique des Plasmas, Paris, 6-10 décembre 1976.
Projection de métaux et de réfractaires pour le traitement de surface, le découpage et la fusion [4, 24, 20] ;

Affinage de matériau par les procédés à l'arc étiré [4], traitement des surfaces polymériques et des fibres [2], synthèse fine par l'oxygène singulet [25] ; dans ces quelques exemples il est déjà possible de classer les applications des plasmas thermiques et des plasmas à excitation plus sélective.

En effet les travaux sur les sources à plasma ont permis une distinction technologique et théorique entre source à effet thermique (arc, torche) et source à excitation sélective (laser, décharge couronne...). 
Toutefois leurs applications au domaine de la production supposent simultanément l'analyse des 4 étapes suivantes :

- l'étape de base est constitué par le choix d'une source à plasma adaptée au problème à traiter,

- la seconde étape réside dans le choix des réactifs et du mélangeage réactifs, fluides plasmagène,

- la troisième étape consiste à définir les paramètres de fonctionnement du réacteur haute température,

- lors de la quatrième étape il s'agira de concevoir un réacteur de trempe adapté à la synthèse du produit recherché.

Pour apporter des éléments de réponse à ces problèmes, il est d'abord nécessaire d'avoir une bonne connaissance des caractéristiques physiques de la décharge (nature des espèces excitées, niveau d'excitation, durée de vie...) et des caractéristiques techniques de son fonctionnement (technologie, limites d'emploi, puissance, rendement énergétique...). En effet la source est un des éléments de l'atelier de fabrication et sa connaissance précisera les caractéristiques de fonctionnement du réacteur haute énergie, lieu de synthèse ou d'élaboration d'espèces chimiques nouvelles (atomes, radicaux ou molécules).

Enfin le réacteur de trempe permet la recombinaison ou la conservation des espèces élaborées dans la zone haute énergie. Ainsi l'association sources à plasma, réacteur haute énergie, réacteur de trempe permet la réalisation d'un procédé adapté à un objectif précis, c'est-à-dire autorisant un taux de conversion et un rendement énergétique aussi élevé que possible.

1. Comparaison entre différentes sources de plasma. - De nombreuses classifications ont été proposées pour différencier les effets des sources à plasma, le tableau I résume les principaux paramètres et propose une distinction physicochimique des différents types de décharge. En premier lieu la pression semble un élément déterminant sur la température des neutres, d'autre part l'énergie contenue par unité de volume varie de façon considérable selon le type de plasma.

- Les plasmas thermiques (torche ou arc) sont considérés comme localement à l'équilibre thermodynamique, c'est-à-dire que le taux d'ionisation est décrit par une réaction de type $\mathrm{A} \rightleftharpoons \mathrm{A}^{+}+\mathrm{e}(\Delta H$ énergie d'ionisation). Aussi est-il possible de calculer à partir de l'équation de Saha la concentration en ion en fonction de la température :

$$
\frac{\left[\mathrm{A}^{+}\right]\left[\mathrm{e}^{-}\right]}{[\mathrm{A}]}=\frac{P_{\mathrm{i}} P_{\mathrm{e}}}{P_{0}}\left[\frac{2 \pi m K}{h^{3}}\right] T^{3 / 2} \exp \left(-\frac{E}{R T}\right) \text {. }
$$

$P_{\mathrm{i}}, P_{\mathrm{e}}, P_{0}$ : poids statiques respectifs de l'ion, l'électron et des particules neutres, $m:$ masse de l'électron,

$k$ : Cte de Boltzmann,

$h:$ Cte de Planck,

$E_{1}$ : énergie d'ionisation du gaz,

$R$ : constante des gaz parfaits.

Connaissant la concentration des ions, la température du gaz et la pression, on peut donc calculer en outre l'enthalpie et la chaleur spécifique disponible par unité de volume.

- Les plasmas froids ou hors d'équilibre sont caractérisés par un écart de température ou plutôt d'énergie important entre les ions, les neutres et les électrons.

La température électronique moyenne peut être estimée à partir de la relation de Von Engel [9] bien que celle-ci s'applique plus particulièrement à des plasmas entre électrodes.

$$
\left|\frac{e V_{\mathrm{i}}}{K T_{\mathrm{e}}}\right|^{1 / 2} \exp \left|\frac{e V_{\mathrm{i}}}{K T_{\mathrm{e}}}\right|=1,16 \times 10^{7}(c p r)^{2}
$$

$V_{\mathrm{i}}$ potentiel d'ionisation

$r$ rayon du tube

$p$ pression

Cte qui dépend de la nature du gaz.

TABleau I

Cas des décharges électriques dans les hydrocarbures

[Electrical discharge in hydrocarbons gases]

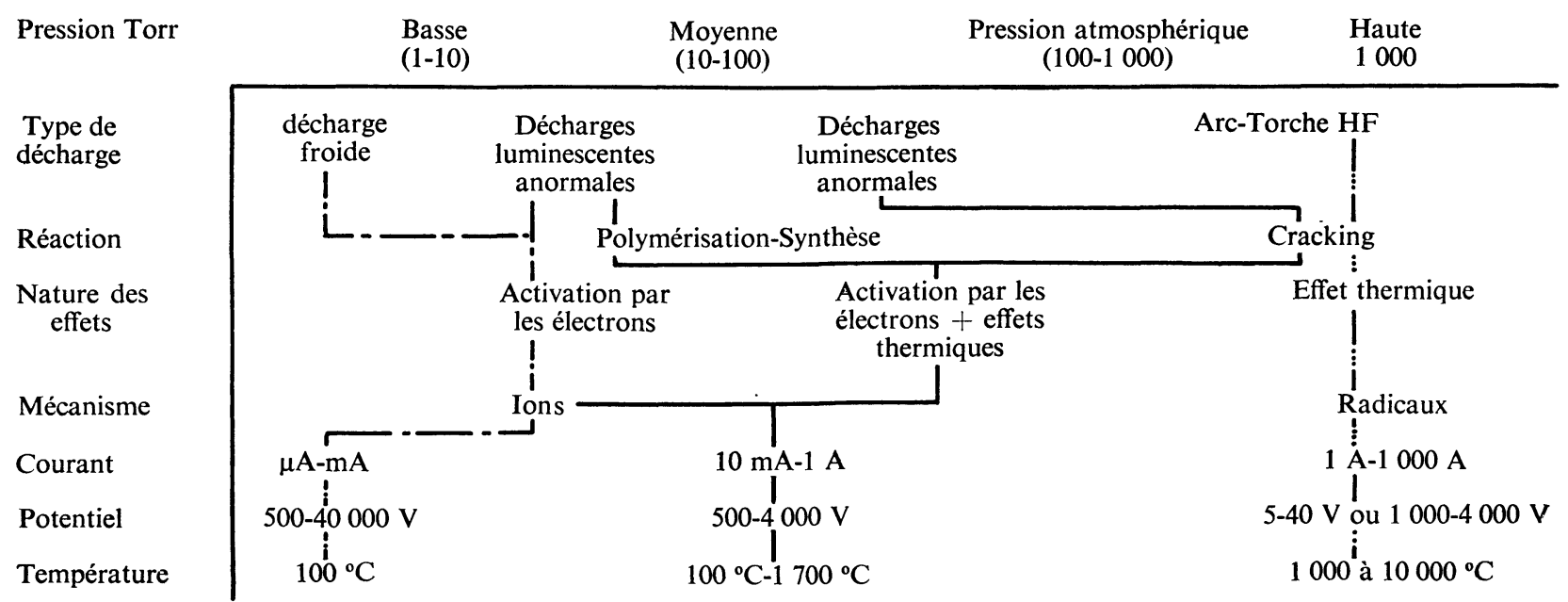


Toutefois la connaissance de la distribution de l'énergie électronique est plus complexe. Druyvestein et Penning ont proposé un type de distribution pour une décharge entre électrode. Toutefois des mesures par sonde peuvent confirmer ou infirmer la théorie choisie.

La température des neutres pourra être mesurée par l'analyse des bandes de vibration et de rotation en spectroscopie.

A partir de cette approche très rapide nous pouvons déjà préciser que le premier type de décharge se caractérisera par une dissociation importante des molécules introduites, le second type de décharge provoquant plutôt une excitation des espèces (excitation électronique, vibrationnelle et rotationnelle). En effet les fluides plasmagènes utilisés en chimie des plasmas sont soit des molécules biatomiques, soit des mélanges de molécules polyatomiques.

Toutefois il est possible d'établir un classement des principales réactions élémentaires parmi toutes celles qui caractérisent une décharge et de leur attribuer un effet chimique (Tableau II). L'importance relative de tel ou tel type de réaction distinguera les sources, leurs implications chimiques et leur rendement énergétique.

Aussi les réactions 1 à 4 distinguent le type de source à plasma employé et la nature du plasmagène utilisé. Les réactions 5 et 6 caractérisent les transformations chimiques dans la zone haute énergie c'est-àdire la zone de synthèse ou de rupture de liaison chimique. Les réactions 7 et 8 caractérisent le réacteur de trempe, c'est-à-dire le processus de récupération d'espèces moléculaires, radicalaires et par conséquent définiront le rendement en produit recherché.

Nous pouvons appliquer ce principe au cas particulier de la synthèse de monoxyde d'azote et constater à partir de la figure 1 que l'on trouve les principales étapes énoncées. L'écart énergétique le plus grand entre l'état final recherché et l'état transitoire correspond au passage par l'état ionisé $(15 \mathrm{eV})$ et en plus il conduit à une décomposition très rapide du monoxyde d'azote dans le réacteur de trempe. Par contre il faut souligner que la synthèse de NO à partir de l'état dissocié de l'azote exige moins d'éner-

\section{TABleau II}

\section{Réactions élémentaires dans une décharge \\ [Elementary reactions in a discharge]}

1. Réactions d'ionisation (dépendant du type de décharge)

photo ionisation $\mathrm{A}+\mathrm{h} v \longrightarrow \mathrm{A}^{+}+\mathrm{e}^{-}$

particules lourdes $\mathrm{A}+\mathrm{B} \longrightarrow \mathrm{A}^{+}+\mathrm{B}+\mathrm{e}^{-}$

2. Réactions de transfert de charge

$$
\mathrm{A}+\mathrm{B}^{+} \longrightarrow \mathrm{A}^{+}+\mathrm{B}
$$

3. Réactions d'attachement

$$
\begin{aligned}
& \mathrm{A}+\mathrm{e}^{-} \longrightarrow \mathrm{A}^{-} \\
& \mathrm{AB}+\mathrm{e}^{-} \longrightarrow \mathrm{A}^{-}+\mathrm{B}
\end{aligned}
$$

4. Réactions d'excitation (mécanismes catalytiques)

photons : $\mathrm{A}+\mathrm{h} v \longrightarrow \mathrm{A}^{*}$

particules : $\mathrm{A}+\mathrm{B} \longrightarrow \mathrm{A}^{*}+\mathrm{B}$

électrons : $\mathrm{e}^{-}+\mathrm{A} \longrightarrow \mathrm{A}^{*}+\mathrm{C}$

transferts d'états excités : $\mathrm{A}^{*}+\mathrm{B} \longrightarrow \mathrm{B}^{*}+\mathrm{A}$

5. Réactions de dissociation (dépendant du fonctionnement du réacteur chaud)

photons : $\mathrm{A}_{2}+\mathrm{h} v \longrightarrow \mathrm{A}+\mathrm{A}$

particules lourdes : $\mathrm{A}_{2}+\mathrm{B} \longrightarrow \mathrm{A}+\mathrm{A}+\mathrm{B}$

élecirons : $\mathrm{A}_{2}+\mathrm{e}^{-} \longrightarrow \mathrm{A}+\mathrm{A}+\mathrm{e}^{-}$

$$
\mathrm{AB}^{*} \longrightarrow \mathrm{A}+\mathrm{B}
$$

6. Réactions ions-molécules (dépendant du fonctionnement du réacteur chaud)

$$
\mathrm{A}^{+}+\mathrm{B} \longleftrightarrow \mathrm{AB}^{+}
$$

7. Réactions de recombinaison (caractérisant le réacteur de trempe)

entre atomes : $\mathrm{B}+\mathrm{A}+\mathrm{A} \longrightarrow \mathrm{B}+\mathrm{A}_{2}$
électrons + ions $\left\{\begin{array}{l}\mathrm{e}^{-}+\mathrm{AB} \longrightarrow \mathrm{A}+\mathrm{B} \\ \mathrm{e}^{-}+\mathrm{A}^{+} \longrightarrow \mathrm{A}+\mathrm{h} v\end{array}\right.$
ions + ions : $\mathrm{A}^{-}+\mathrm{B}^{+} \longrightarrow \mathrm{AB}$
radicaux : $\mathrm{R}^{\prime}+\mathrm{H} \longrightarrow$ polymères
molécules : monomères $\longrightarrow$ PH




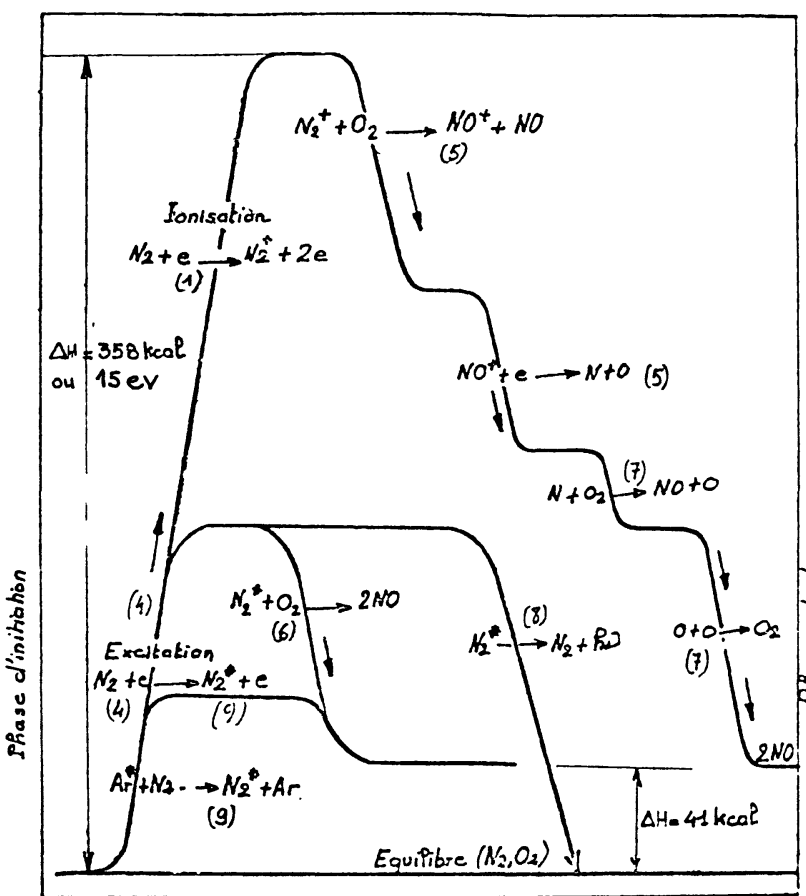

SYNTHESE DE NO

FIG. 1. - Synthèse de NO.

[NO synthesis.]

gie $(10 \mathrm{eV})$ (Fig. 2), enfin si l'on admet qu'une partie de monoxyde est produit par réaction entre l'azote moléculaire excité $(6 \mathrm{eV})$ et la molécule d'oxygène, l'énergie minimale requise pour initier la réaction est alors plus faible. Ainsi le rendement énergétique du réacteur varierait selon le cas retenu entre $12 \%$ et $55 \%$ d'énergie fixée par la réaction chimique.

Cette même analyse s'applique à l'exploitation industrielle de fabrication de l'acétylène pour lequel le taux d'énergie fixé par la synthèse est de $35 \%$ à

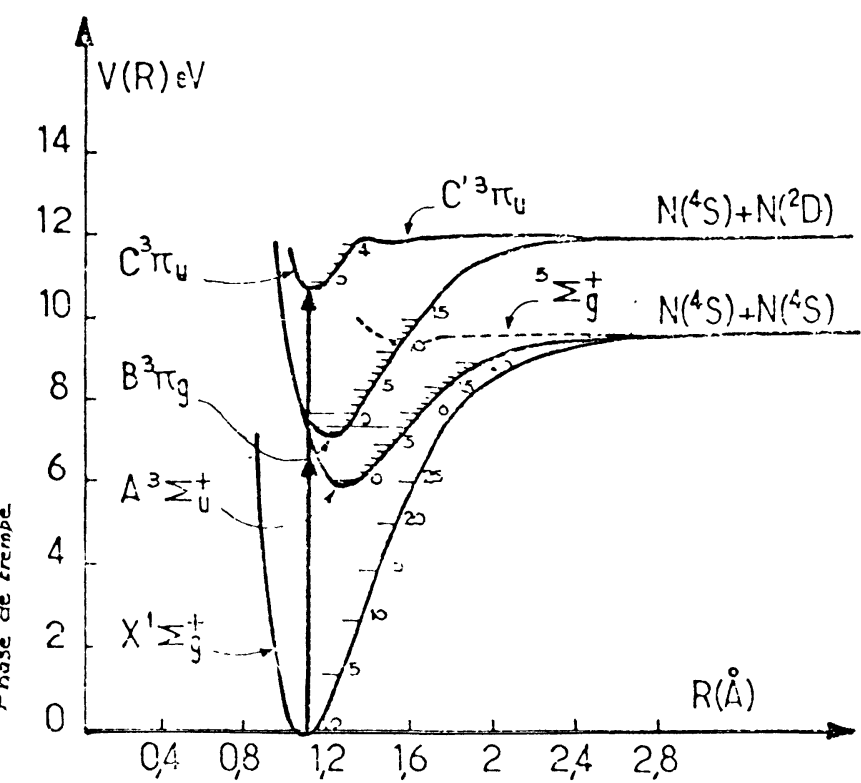

Fig. 2. - Courbes d'énergie potentielle et niveaux vibrationnels de $\mathrm{N}_{2}$ selon Gilmore. La flèche verticale indique les transitions à partir du niveau $\mathrm{X}^{1} \Sigma_{\mathrm{g}}^{+}, v=0$ suivant le principe de FranckCondon.

[Curves of potential energy and vibrational levels for $\mathrm{N}_{2}$ molecule.]

$50 \%$ de l'énergie contenue dans le plasma selon l'énergie récupérée dans la phase de trempe et le niveau d'injection des réactifs.

Dans ces conditions afin de satisfaire au double impératif du rendement chimique et du rendement thermique, l'ingénieur devra identifier les combinaisons de réactifs qui favorisent les transferts d'énergie dans la source à plasma et dans le réacteur, tout en améliorant le rendement chimique. Enfin des considérations d'ordre économiques pourront être également retenues pour le choix de la source tel que le prix du kWh (Tableau III).

\section{TABleau III}

Comparaison des différents type de générateurs [Comparison of different plasma generators]

\begin{tabular}{|c|c|c|c|c|}
\hline Type & $\begin{array}{c}\text { DC-Arc } \\
-\end{array}$ & $\begin{array}{c}\text { 3-Phase AC-Arc } \\
-\end{array}$ & Torche inductive & Film liquide DC-Arc \\
\hline Puissance & $150-200 \mathrm{~kW}$ & $500-1000 \mathrm{~kW}$ & $100-1000 \mathrm{~kW}$ & $300-500 \mathrm{~kW}$ \\
\hline Prix $\mathrm{kW}$ installé en $\mathrm{F}$ & $1400 \mathrm{~F} / \mathrm{kW}$ & $1600 \mathrm{~F} / \mathrm{kW}$ & $\begin{array}{l}3500-5000 \mathrm{~F} / \mathrm{kW} \\
1000-1250 \mathrm{~F} / \mathrm{kW}\end{array}$ & $1500 \mathrm{~F} / \mathrm{kW}$ \\
\hline Gaz utilisé & gaz nobles $\mathrm{N}_{2}$ & gaz nobles $\mathrm{N}_{2}$ & $\begin{array}{l}\text { mélangeages gazeux } \\
\text { quelconques }\end{array}$ & $\begin{array}{l}\text { tous les liquides } \\
\text { même isolant, eau, } \\
\text { alcool, hydrocar- } \\
\text { bures }\end{array}$ \\
\hline Nature de l'atmosphère & $\begin{array}{l}\text { neutre ou réduc- } \\
\text { trice }\end{array}$ & $\begin{array}{l}\text { neutre ou réduc- } \\
\text { trice }\end{array}$ & $\begin{array}{l}\text { neutre, réductrice, } \\
\text { oxydante, réactive }\end{array}$ & $\begin{array}{l}\text { neutre, réductrice, } \\
\text { oxydante }\end{array}$ \\
\hline $\begin{array}{l}\text { Rendement du générateur } \\
\text { Rendement de la source }\end{array}$ & $\begin{array}{l}60-80 \% \\
90-95 \%\end{array}$ & $\begin{array}{c}80 \%+ \\
95 \%\end{array}$ & $\begin{array}{l}50 \%+ \\
30-80 \%\end{array}$ & $\begin{array}{l}85-90 \% \\
90-95 \%\end{array}$ \\
\hline
\end{tabular}


SOURCE A PLASMA ET PROCESSUS CHIMIQUES

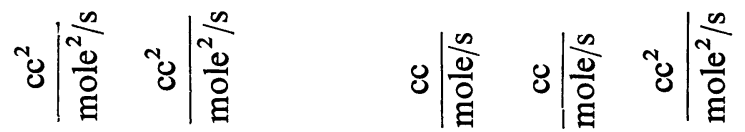

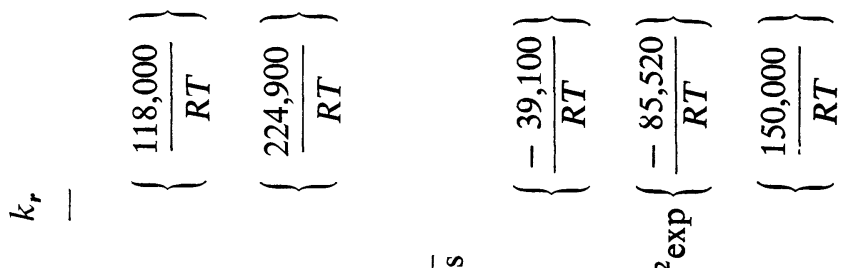

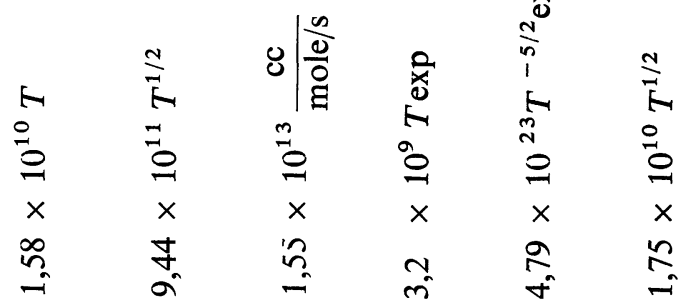

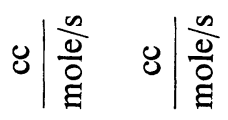

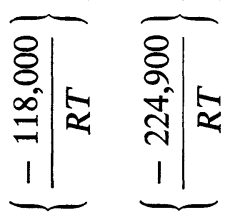

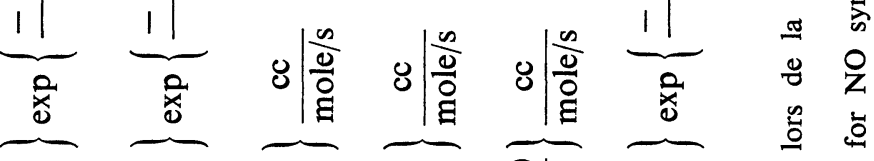

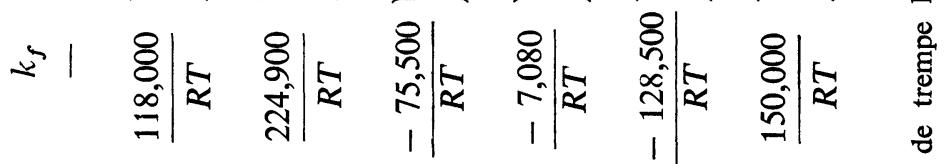

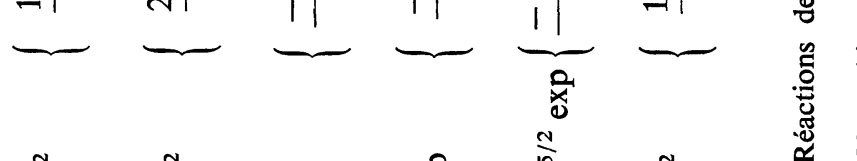

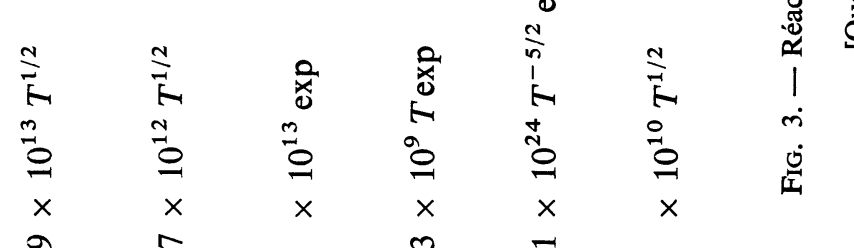

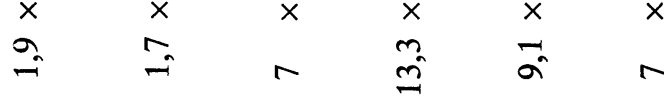

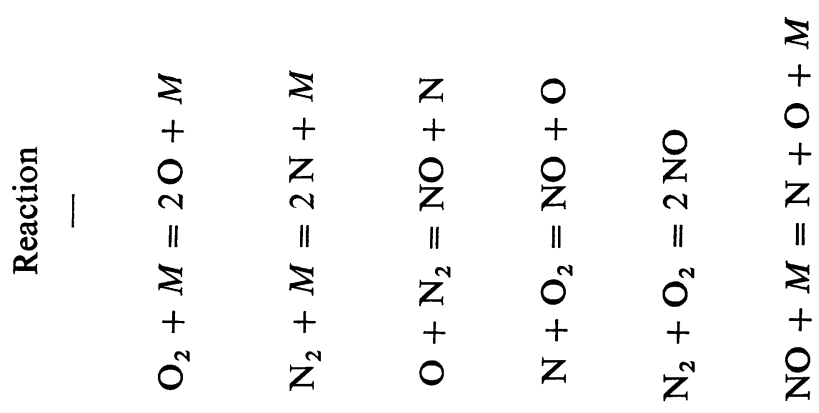

$$
\begin{aligned}
& \begin{array}{llllll}
2 & \nabla & 0 & \infty & 0 & \approx \\
-\pi & n & n & 0 & -1
\end{array}
\end{aligned}
$$


2. Analyse des paramètres de fonctionnement du réacteur de synthèse. - Compte tenu des mécanismes exposés dans le tableau II, nous distinguerons 3 étapes dans le réacteur de fabrication :

2.1 LA SOURCE A PLASMA ET LES PARAMÈTRES TECHNIQUES RETENUS. - Caractéristique de la décharge électrique ( $V, I$ fréquence, nombre de phase) ;

- Nature des électrodes (W, Cu...) ;

- Géométrie des électrodes (pointe plaque, cylindrique...) ;

- Critère de stabilisation de la décharge (vortex, champ magnétique) ;

- Nature des parois et géométrie adoptée ;

- Pression de travail, densité de charges, nature des ions et des espèces excitées ;

- Rendement énergétique de ce type de source.

2.2 PARAMÈTRES DE FONCTIONNEMENT DU RÉACTEUR HAUTE ÉNERGIE. - Nous tenterons de le définir par une analyse [21, 22] voisine de celle des réacteurs classiques :

2.2.1 Paramètres de mélangeage pour les réacteurs en phase homogène et hétérogène. - Mélangeage des réactifs avant leur introduction dans le plasma;

- Choix d'un fluide plasmagène distinct des réactifs ;

- Présence de particules solides dans le réacteur;

- Injection de l'un des réactifs en aval.

2.2.2 Paramètres énergétiques. - Transfert d'énergie entre le fluide plasmagène et les réactifs (tant pour un système homogène qu'un système hétérogène) ;

- Transfert d'énergie à la paroi. Recherche d'une paroi chaude, à film liquide... ;

- Calcul du rendement énergétique du réacteur.

2.2.3 Paramètres chimiques. - Identification des réactions chimiques principales et secondaires susceptibles d'apparaître selon les espèces excitées présentes ;

- Recherche de l'obtention d'un état excité [25] spécifique pour l'un des réactifs (oxygène singulet);

- Recherche d'espèces excitées à basse température pour les traitements de polymères en surface [2] ;

- Calcul du temps de séjour dans le réacteur à haute énergie ;

- Calcul du rendement chimique.

2.3 PARAMÈTRES DE FONCTIONNEMENT DU RÉACTEUR DE TREMPE. - Il s'agit dans cette $3^{\mathrm{e}}$ étape de récupérer les espèces radicalaires atomiques ou moléculaires produites dans la zone haute température et qui représentent le produit recherché. Il s'agira donc d'éviter leur destruction en permettant les recombinaisons et le transfert d'énergie. On distinguera les cas suivants:

2.3.1 Récupération du produit formé dans le réacteur haute température. - Il y aura compétition entre la cinétique de dégradation du produit formé et la vitesse de transfert d'énergie à la paroi. Exemple trempe de l'acétylène par film d'eau [15].

2.3.2 Recombinaison de radicaux [Réf. 1]. - Réaction des radicaux dans le liquide de trempe :

$$
\mathrm{NH}+\mathrm{NH}_{3 \text { liquide }} \rightarrow \mathrm{NH}_{2}-\mathrm{NH}_{2} \text {. }
$$

- Réaction de dégradation

$$
\mathrm{NH}_{2}-\mathrm{NH}_{2}+\mathrm{NH} \rightarrow \mathrm{NH}_{3}+\mathrm{N}_{2}+\mathrm{H}_{2} \text {. }
$$

- Cinétique de transfert d'énergie vers la paroi.

2.3.3 Réaction chimique dans le réacteur de trempe [1, 10, 15] (Fig. 3). - Réaction entre espèces excitées et espèces dans l'état fondamental.

- Réaction entre $\mathrm{N}_{2}$ et $\mathrm{N}$ et l'oxygène moléculaire utilisé pour la trempe.

- Réaction de désexcitation.

- Réaction de dégradation de NO.

- Réaction de transfert d'énergie à la paroi.

A partir de ces trois étapes il est possible de définir un rendement chimique et énergétique global.

3. Exemples d'application dans le cas de la synthèse chimique en phase homogène. - 3.1 RôLE DE L'ÉTUDE THERMODYNAMIQUE. - Elle permettra pour un plasma proche de l'E. T. L. de définir dans une première approche un certain nombre de paramètres techniques qui conditionnent la marche du réacteur haute température, en particulier la nature des réactifs à employer, leur proportion, la pression de travail, la température moyenne à retenir et la zone d'introduction.

Pour présenter cette étude nous avons choisi 2 exemples :

3.1.1 Etude du système C-H-N. - Le diagramme d'équilibre du système $\mathrm{C}-\mathrm{H}-\mathrm{N}$ permet d'identifier les domaines de formation de l'acétylène et de l'acide cyanhydrique en fonction de la composition du mélange de base et de la pression de travail.

La figure 4 indique clairement que l'acétylène est l'espèce carbonée majoritaire $(70 \%$ du carbone introduit) vers $1300 \mathrm{~K}$, sa présence étant d'ailleurs facilité par la présence d'azote qui permet l'apparition de l'acétylène à une température $200 \mathrm{~K}$ plus basse qu'en l'absence d'azote. Par contre vers $2500 \mathrm{~K}$ l'acide cyanhydrique est l'espèce carbonée majoritaire $(60 \%$ du carbone introduit).

Les diagrammes ternaires $\mathrm{C}-\mathrm{H}-\mathrm{N}$ ont été tracés pour les espèces carbonées majoritaire présentes à l'équilibre, ils indiquent le rôle de la température et $\mathrm{du}$ rapport $\mathrm{N} / \mathrm{C}$ sur la nature et la concentration en produits formés. Ces informations permettent d'identifier le rôle des paramètres physicochimiques qui contrôlent le réacteur haute təmpérature. En plus elles indiquent la nature des radicaux qui devront se recombiner lors de la trempe si la température du réacteur dépasse $2900 \mathrm{~K}$ (Fig. 5) : $\mathrm{C}_{2} \mathrm{H}$ et $\mathrm{CN}$. 


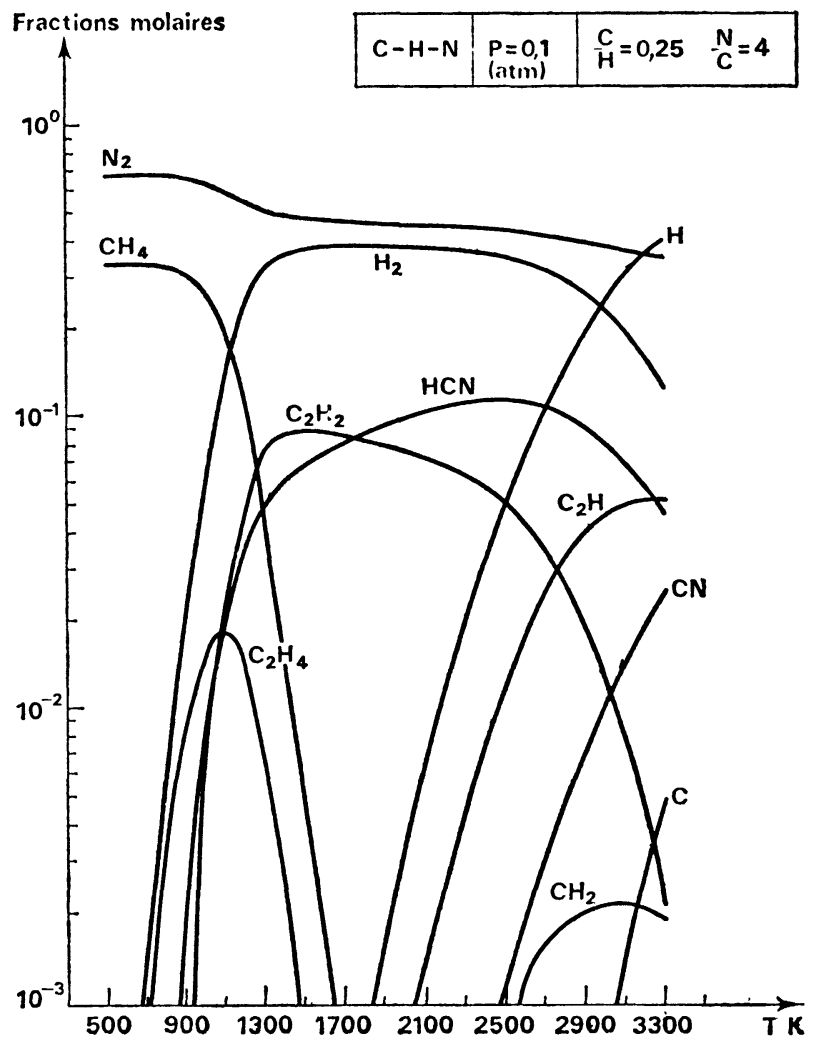

Fig. 4. - Diagramme d'équilibre du système C-H-N.

[Equilibrium diagram for C-H-N system.]

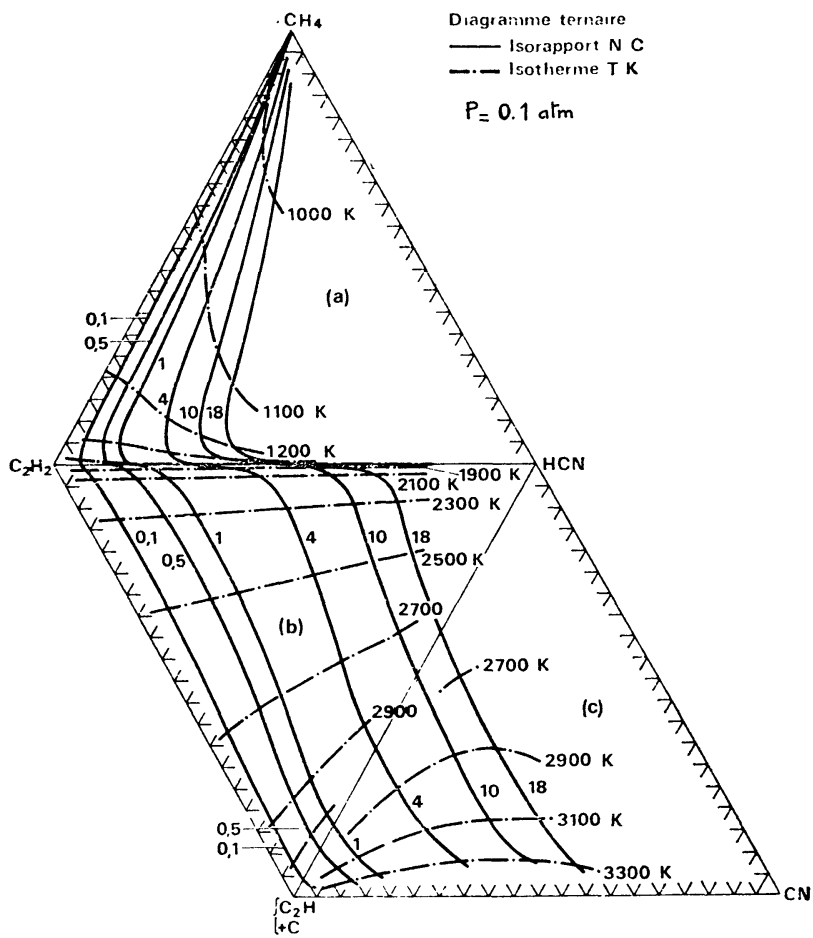

Fig. 5. - Diagramme ternaire.

[Ternary diagram.]

L'ensemble de ces études ont été réalisées à la pression de 0,1 atm, celle-ci n'ayant d'ailleurs qu'un rôle négligeable comme le montre une étude plus complète [29].
3.1.2 Etude du système N-O. - Les diagrammes d'équilibre réalisés pour un rapport équimolaire $\mathrm{N}_{2} / \mathrm{O}_{2}$ indiquent nettement le rôle de la piession sur la teneur en NO formé. Toutefois deux domaines sont considérés selon que l'on considère le réacteur haute température comme le réacteur de formation de NO, ou celui de formation de l'azote dissocié (Fig. 6).

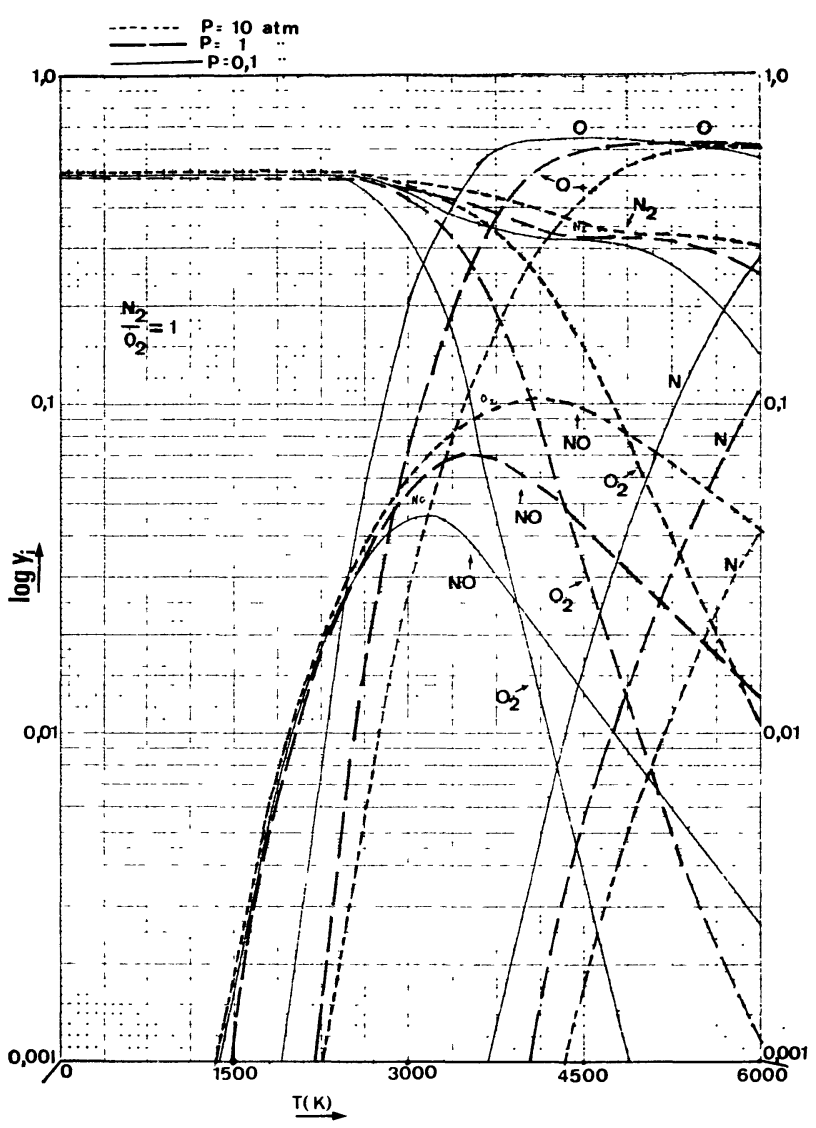

FIG. 6. - Diagramme d'équilibre du système N-O.

[Equilibrium diagram for $\mathrm{N}-\mathrm{O}$ system.]

Dans la première hypothèse la teneur maximale possible en oxyde d'azote n'excède pas $10 \%$ pour $P=10 \mathrm{~atm}$ à $(4200 \mathrm{~K})$ de la teneur en azote introduit et la pression a un rôle favorable sur la fixation de l'azote. Dans le second cas la teneur maximale possible en azote dissocié sera voisine de $30 \%$ à $6000 \mathrm{~K}$ et $P=0,1$ atm et la pression à un effet défavorable. Toutefois la teneur en NO formée ne sera égale à la teneur en azote dissocié que si le réacteur de trempe permet une conversion totale de l'azote dissocié en NO.

Dans ces conditions nous pouvons déjà conclure que la torche à plasma d'azote avec injection en aval de la tuyère d'un débit d'oxygène en quantité identique devra constituer la meilleure solution de travail, la pression étant le paramètre à étudier. Enfin nous pouvons améliorer le rendement de la source en utilisant le transfert argon-azote afin d'augmenter le nombre d'états excités susceptibles de fournir une dissociation de $\mathrm{N}_{2}$ et diminuer parallèlement le nombre 
d'atomes ou de molécules d'azote ionisés qui sont de grands consommateurs d'énergie par molécule d'azote.

3.2 DÉFINITION DU RÉACTEUR HAUTE TEMPÉRATURE DANS LE CAS DE LA TRANSFORMATION $\mathrm{CH}_{4}-\mathrm{C}_{2} \mathrm{H}_{2}$. L'étude réalisée consistait à étudier le rôle du temps de séjour d'un mélange méthane-argon dans des réacteurs graphite de longueur différente.

Afin de confronter les résultats expérimentaux avec ceux de la théorie, nous avons supposé que le réacteur pouvait être caractérisé par un mélangeage immédiat argon-méthane compte tenu de la présence d'un effet de vortex dans la zone d'introduction du méthane. Pour le bilan thermique nous avons admis que le réacteur fonctionne de façon adiabatique, et le jeu de réactions chimiques pris en considération est le suivant :

$$
\begin{array}{r}
2 \mathrm{CH}_{4} \rightarrow \mathrm{C}_{2} \mathrm{H}_{2} k_{1}=10^{12} \exp \left(\frac{80000}{R T}\right), \\
\Delta H_{1}=90000 \mathrm{cal}, \\
\mathrm{CH}_{4} \rightarrow \mathrm{C}+2 \mathrm{H}_{2} k_{2}=3,6 \times 10^{3} \exp \left(\frac{16200}{R T}\right), \\
\Delta H_{2}=18000 \mathrm{cal}, \\
\mathrm{C}_{2} \mathrm{H}_{2} \rightarrow 2 \mathrm{C}+\mathrm{H}_{2} k_{3}=1,8 \times 10^{4} \exp \left(\frac{27500}{R T}\right), \\
\Delta H_{3}=-54000 \mathrm{cal} .
\end{array}
$$

Les résultats obtenus expérimentalement et par le calcul simplifié montrent un bon accord entre la théorie et l'expérience (Figs. 7, 8, 9), ils indiquent dans ce cas précis que le temps de séjour n'est pas un paramètre déterminant pour la teneur en acétylène car la composition du mélange est à l'équilibre. Toutefois une augmentation du temps de séjour se

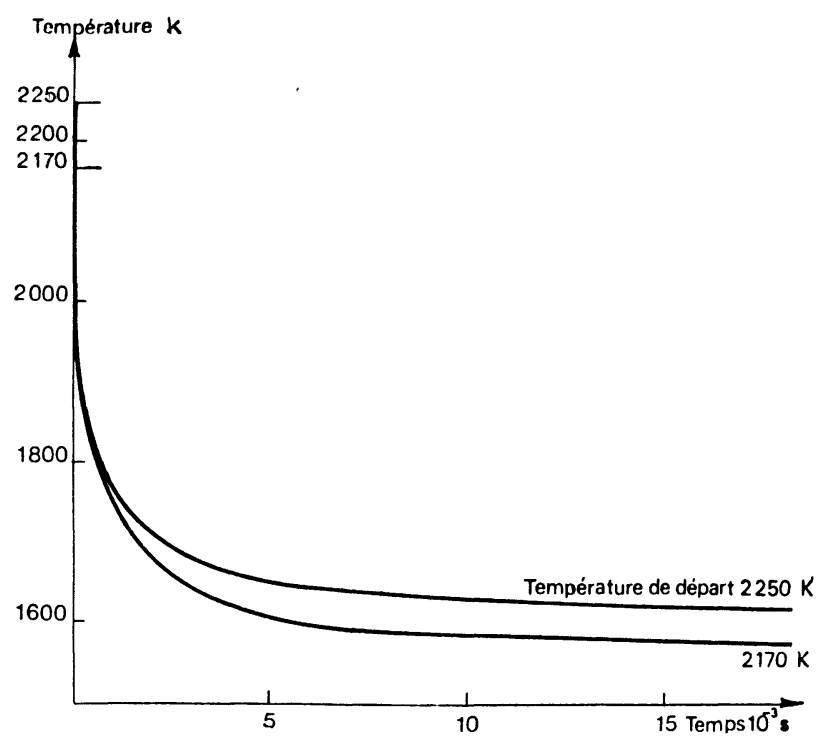

FIG. 7. - Variation de la température dans les réacteurs. [Temperature variation as a function of residence time (data results).]

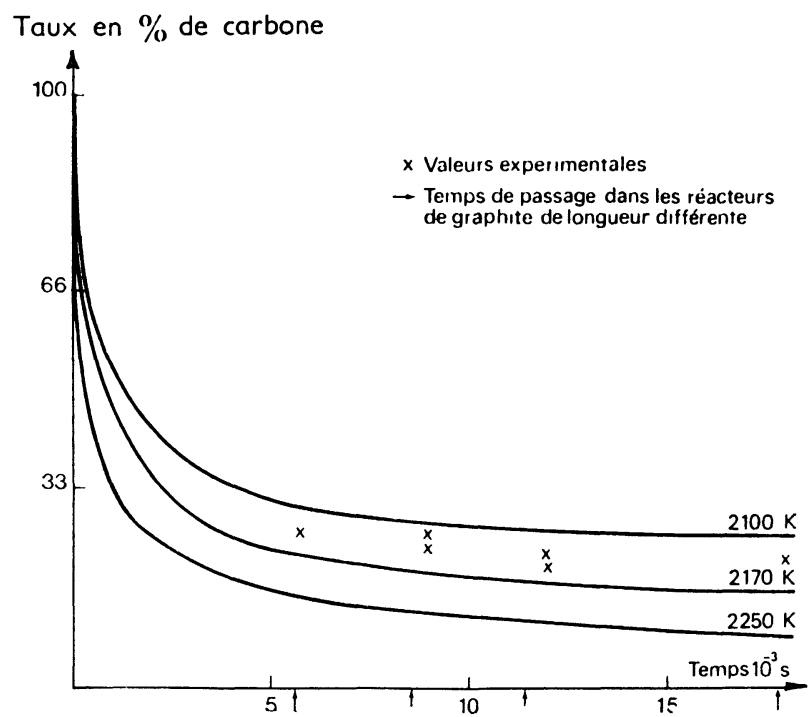

FIG. 8. - Variation du taux de méthane en fonction du temps. [Methane rate as a function of residence time (data results).]

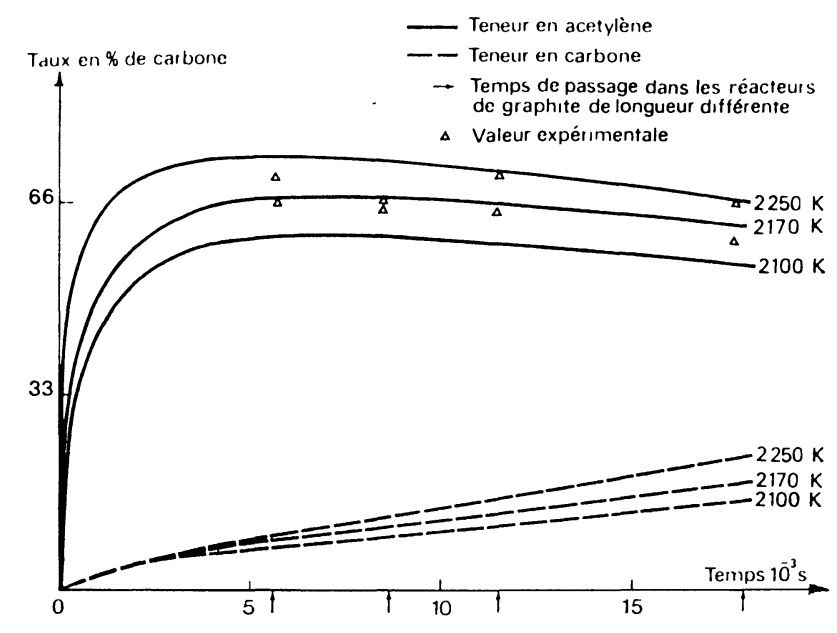

Fig. 9. - Courbe calculée. Variation du taux d'acétylène et de carbone en fonction du temps.

[Acetylene rate and carbon rate as a function of residence time.]

traduit par une augmentation de la teneur en carbone formé, le modèle devient alors inexact du point de vue thermique et les réactions de dépôt de carbone à la paroi l'emportent sur les réactions de synthèse.

3.3 DÉFINITION DU RÉACTEUR DE TREMPE. 3.3.1 Rôle de l'hydrogène lors de la trempe par lit fluidisé [26, 27, 28]. - L'étude de la trempe par lit fluidisé (vitesse de trempe $10^{6} \mathrm{~K} / \mathrm{s}$ ) d'un mélange $\mathrm{CH}_{4}$-argon indique le rôle déterminant joué par l'hydrogène sur la teneur en acétylène produit à la sortie du réacteur. Pour ces essais les caractéristiques du plasma sont restées identiques (argon 23,51/min., méthane $31 / \mathrm{min} ., \quad P=2,5 \mathrm{~kW} / \mathrm{h}$ ) (Fig. 10,11 ).

Dans ces conditions nous constatons que l'augmentation de la teneur en hydrogène provoque une varia- 


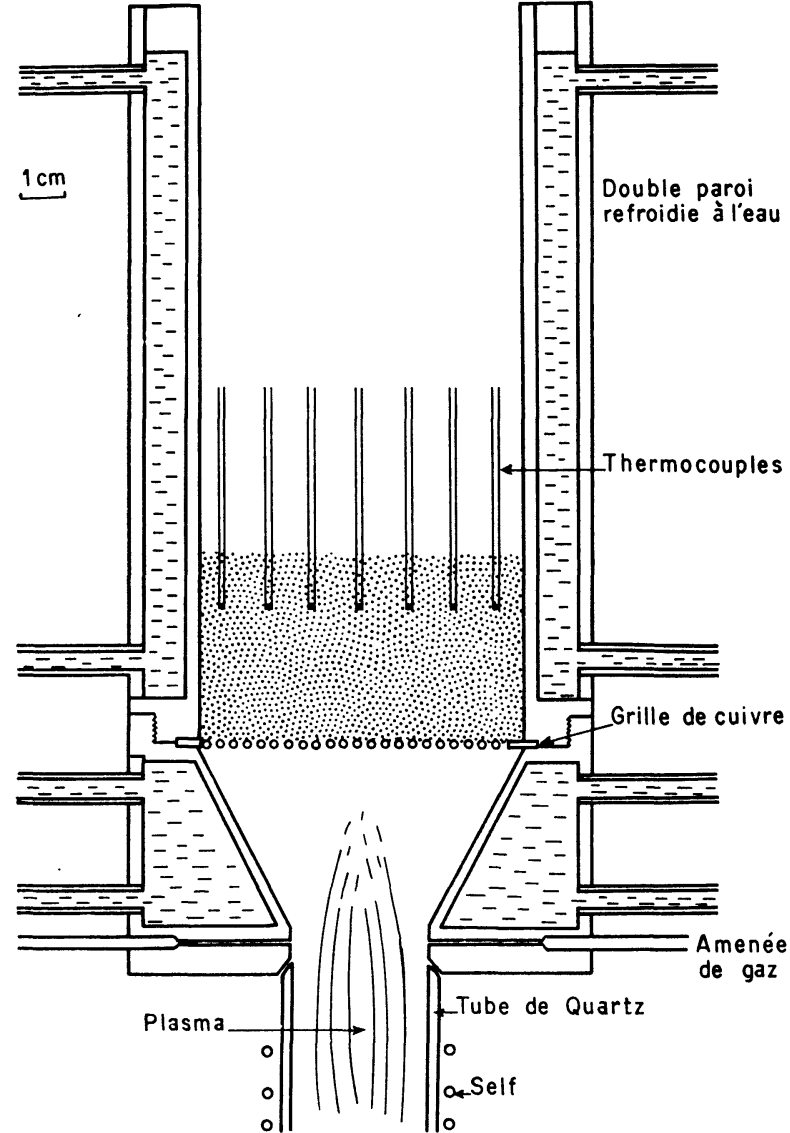

Fig. 10. - Trempe par lit fluide.

[Fluidized bed quenching reactor.]

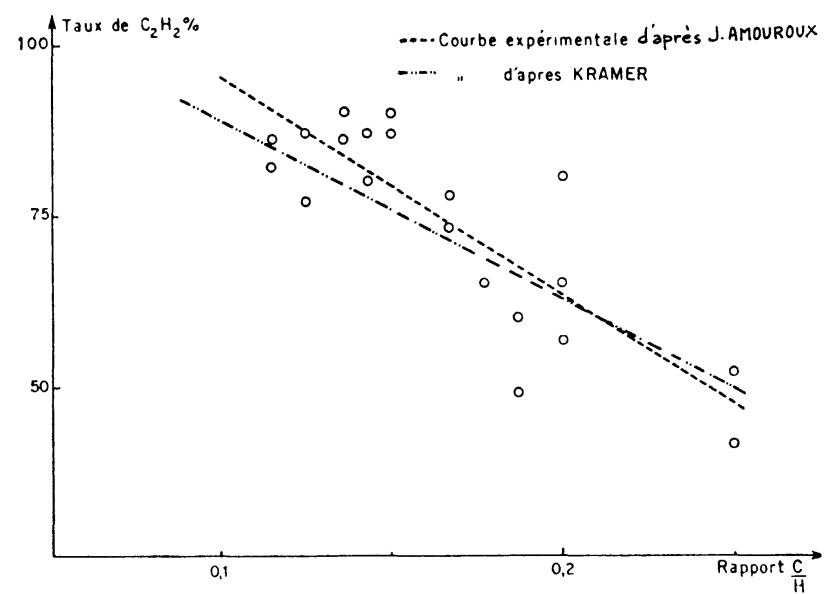

FIG. 11. - Rendement en acétylène en fonction du rapport $\mathrm{C} / \mathrm{H}$. [Acetylene rate as a function of $\mathrm{C} / \mathrm{H}$ ratio.]

tion de la teneur en acétylène de 50 à $90 \%$ et diminue la formation de noir de carbone.

Ce phénomène s'interprête de 2 façons : diminution du nombre de molécules de carbone vapeur formées dans le réacteur chaud et hydrogénation des radicaux $\mathrm{C}_{2} \mathrm{H}$ lors de la phase de trempe.

Cette technique est utilisée dans les procédés industriels de fabrication d'acétylène au plasma.
3.3.2 Modèle d'un réacteur de trempe à l'eau [15, 27]. - La vaporisation d'un film d'eau à contre courant d'un plasma d'argon-acétylène est une technique de trempe classique. Ainsi les procédés industriels Montedison ont employé l'injection d'eau, et ceux de la Dupont de Nemours des pulvérisations d'huile [30].

Un modèle du réacteur de trempe consiste à tenir compte de la réaction chimique

$$
\begin{gathered}
\mathrm{C}_{2} \mathrm{H}_{2}+\mathrm{H}_{2} \mathrm{O} \rightarrow{ }_{2} \mathrm{CO}+3 \mathrm{H}_{2}, \\
\Delta H_{\mathrm{i}}=8000 \mathrm{cal} / \mathrm{mole}
\end{gathered}
$$

de la réaction de vaporisation de l'eau

$$
\mathrm{H}_{2} \mathrm{O} \text { liq } \rightarrow \mathrm{H}_{2} \mathrm{O} \text { vap }, \quad \Delta H_{2}=10800 \mathrm{cal} / \mathrm{mole}
$$

du bilan matière et du bilan thermique (annexe 1).

Dans ces conditions, les expériences et la simulation du modèle du réacteur de trempe indiquent nettement que la teneur en acétylène restant dépend essentiellement de la vitesse de trempe, c'est-à-dire de la

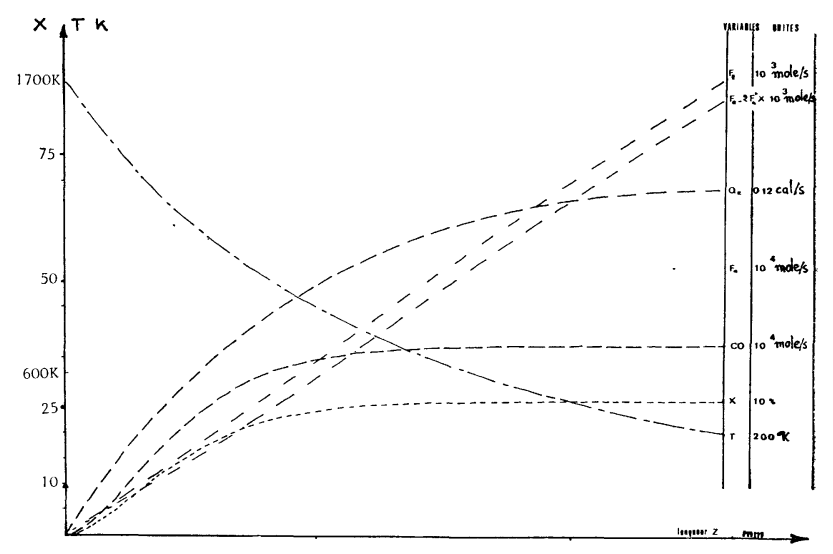

FIG. 12. - Profil de marche du réacteur-débit d'eau : $1055 \mathrm{~cm}^{3} / \mathrm{min}$.

[Simulation of quenching reactor - water flow $1055 \mathrm{~cm}^{3} / \mathrm{min}$.]

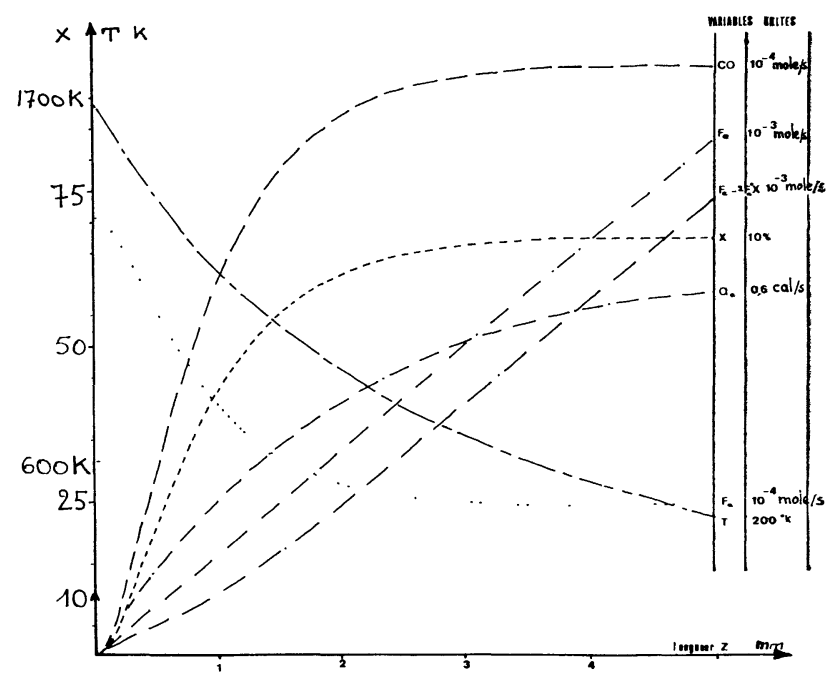

Fig. 13. - Profil de marche du réacteur-débit d'eau : $500 \mathrm{~cm}^{3} / \mathrm{min}$.

[Simulation of quenching reactor - water flow $500 \mathrm{~cm}^{3} / \mathrm{min}$.] 


\section{TABleauX IV ET V \\ Résultats expérimentaux pour le réacteur de trempe \\ [Experimental results for quenching reactor]}

Réacteur de longueur : $5 \mathrm{~cm}$

Diamètre : $2 \mathrm{~cm}$

Débit d'eau
$\% \mathrm{C}_{2} \mathrm{H}_{2}$ formé
$\% \mathrm{CO}$ formé
$\% \mathrm{C}_{2} \mathrm{H}_{4}$ formé
$\% \mathrm{CH}_{4}$ restant
Énergie disponible dans la zone de
mélangeage $(\mathrm{cal} / \mathrm{min}$.)
Débit d'eau $\mathrm{cm}^{3} / \mathrm{min}$.
Temps contact $\mathrm{s}$
Taux de temps $\mathrm{s} / \mathrm{K}$
Vitesse de refroidissement $\mathrm{K} / \mathrm{s}$

$1055 \mathrm{~cm}^{3} / \mathrm{min}$.
$-\quad 48$
17
2
27

17200

500

$7,3 \times 10^{-4}$

$6,1 \times 10^{-4}$

$1,6 \times 10^{+6}$

Débit d'argon : 23 1/min.

Débit de méthane : $3 \mathrm{l} / \mathrm{min}$.

Puissance du plasma : $23000 \mathrm{cal} / \mathrm{min}$.

compétition entre transfert thermique et réaction de combustion dans la vapeur d'eau. Ainsi le taux de conversion en acétylène varie de 50 à $20 \%$ lorsque la vitesse de trempe varie de $7 \times 10^{6}$ à $1,5 \times 10^{6} \mathrm{o} C / \mathrm{s}$ (Figs. 12, 13, Tableaux IV et V).

Par ces quelques exemples nous proposons une méthode pour réaliser une étude complète source de plasma-réacteur haute énergie et réacteur de trempe qui permette de préciser le meilleur rendement chimique et énergétique c'est-à-dire qui optimise l'ensemble.

Cette présentation pose de façon plus précise l'importance du choix de la source pour la réalisation

d'un procédé de fabrication. Toutefois il est nécessaire de la compléter par quelques considérations économiques dans le cas de procédés de synthèses endothermiques au plasma.

4. Coût de fabrication dans le cas d'un procédé au plasma. - L'analyse du coût s'effectue brièvement en 2 étapes, la première consiste à étudier le réacteur et la seconde l'atelier de fabrication.

4.1 LE RÉACTEUR DE FABRICATION. - Son étude consistera à effectuer une analyse des coûts à chaque étape étudiée précédemment.

\begin{tabular}{lll}
\multicolumn{1}{c}{ Bilan financiers } & \multicolumn{1}{c}{ Bilan chimique } & Bilan thermique \\
investissement & choix de la source & rendement énergétique \\
coût des matières premières & choix des réactifs et du fluide plas- & \\
& magène \\
coût d'investissement + entre- & $\begin{array}{c}\downarrow \\
\text { réacteur (configuration, alimenta- } \\
\text { tion-taux de conversion) }\end{array}$ & bilan énergétique partiel \\
coût des fluides de trempe & $\begin{array}{c}\downarrow \\
\text { réacteur de trempe (alimenta- } \\
\text { tion, taux de conversion) }\end{array}$ & bilan énergétique \\
coût global & rendement chimique &
\end{tabular}

Cette étude nous amènera donc à définir un coût en $\mathrm{kWh} / \mathrm{kg}$ de produit pour un investissement donné (Tableau VI).

4.2 Atelier DE fABRicAtion. - L'atelier de fabrication comprendra outre le réacteur toutes les unités de prétraitement des réactifs et d'affinage des produits, les aires de stockage, les unités de contrôle, les unités de traitement des eaux et fumés, etc.... Une étude désormais complète permet une comparaison entre les procédés (Tableau VII) [23].
5. Conclusion. - Le choix de la source de plasma et la connaissance précise de ses caractéristiques physiques (nature des espèces excitées produites, niveaux d'excitation, durée de vie, etc...), et des caractéristiques techniques de la source (conditions de fonctionnement, limites d'emploi, rendement énergétique) permettent de réaliser une installation de transformation chimique adaptée à l'objectif retenu. Toutefois elle exige également une parfaite connaissance des problèmes posés au niveau du calcul des réacteurs haute température et réacteurs de trempe 
TABLEAU VI

Performances des différents réacteurs à arc pour la production d'acétylène

[Performance of various arc acetylene reactors]

$\begin{array}{lcccc} & \text { Huels I } & \text { Huels II } & \text { Hoechst AC } & \begin{array}{c}\text { DuPont de } \\ \text { Nemours }\end{array} \\ \text { V } & 7 \overline{0} & 7100 & 1400 & 3500 \\ \text { A } & 1200 & 4200 & 3 \times 4200 & 3100 \\ \text { MW } & 8,5 & 8,5 & 10,0 & 10,8 \\ \text { Pression } & 1 & 1 & 1 & 0,5 \\ \text { Flux de l'hydrocarbure à travers l'arc } & 1410 & 0 & 0 & 1900 \\ \quad(\mathrm{~kg} / \mathrm{h}) & 1000 & 3200 & 2000 & 0 \\ \text { Flux d'hydrocarbure dans le jet (kg/h) } & 2150 & 1400 & 1470 & 3200 \\ \text { Flux de } \mathrm{H}_{2} \text { recyclé } & 850 & 850 & 1000 & 1240 \\ \mathrm{C}_{2} \mathrm{H}_{2} \text { produit }(\mathrm{kg} / \mathrm{h}) & 10 & 10 & 10 & 6,4 \\ \text { Energie consommée }(\mathrm{kWh} / \mathrm{kg}) & & & & \end{array}$

\section{TABLEAU VII}

Données économiques

[Economic data for different acetylene processes]

Procédé arc-hydrogène

et charbon

pour une production

de $136000 \mathrm{t} /$ an $\mathrm{C}_{2} \mathrm{H}_{2}$ gaz recyclé et trempé.

On obtient

des sous-produits

noir de carbone, $\mathrm{HCN}$

Investissement

Matériau traité

Dépenses préalables globales

Matières premières et matières annexes

Utilités

Vente, recherche intérêt du capital

de roulement

Bilan

Valeur des sous-produits

Coût net de production

Intérêt sur les investissements du capital

Coût net de production

Données $\$ / \mathbf{L b}$.

\section{$53 \times 10^{6} \$$ \\ Charbon}

4,42

1,11

3,42

1,21

10,16

3,82

6,34

1,21

$7,55 \$ / \mathrm{Lb}$
Procédé qui utilise

l'oxydation partielle du $\mathrm{CH}_{4}$

pour une production de $136000 \mathrm{t}$ /an

$$
40 \times 10^{6} \$
$$

Méthane

2,64

9,00

0,09

1,20

12,93

3,69

9,24

0,91

$10,15 \$ / \mathrm{Lb}$
Procédé qui utilise l'oxydation partielle de Napht.

Production 136000 t/an

$32 \times 10^{6} \$$

Naphta

2,31

4,42

0,41

0,61

7,95

7,95

0,73

$8,68 \$ / \mathrm{Lb}$ afin de permettre la mise au point d'un procédé nouveau. Nous pouvons conclure en disant qu'il n'y a pas de source de plasma à applications universelles, chaque mise en œuvre doit répondre à un objectif pour optimiser le procédé de fabrication.

\section{Signification des symboles}

$\begin{array}{ll}\mathrm{a} & : \text { acétylène. } \\ C & : \text { capacité calorifique molaire (cal/mole). } \\ C_{\mathrm{g}} & : \text { coefficient de transfert à la paroi. }\end{array}$ e $\quad:$ eau.

$F(\mathrm{i}, \mathrm{e}, \mathrm{a}, \mathrm{t})$ : flux molaire de i, e, a, t (mole/s).

$\Delta H \quad$ : enthalpie de combustion de l'acétylène cal/mole.

$\Delta H^{\prime} \quad$ : enthalpie de vaporisation de l'eau cal $/$ mole.

$H_{\mathbf{y}} \quad$ : indice hydrogène.

i : constituants gazeux initiaux.

j : indice des constituants du mélange (acétylène, hydrogène, $\mathrm{CO}$...).

: constante cinétique de la réaction $1 \mathrm{~mole}^{-6} / \mathrm{s}^{-1}$. 

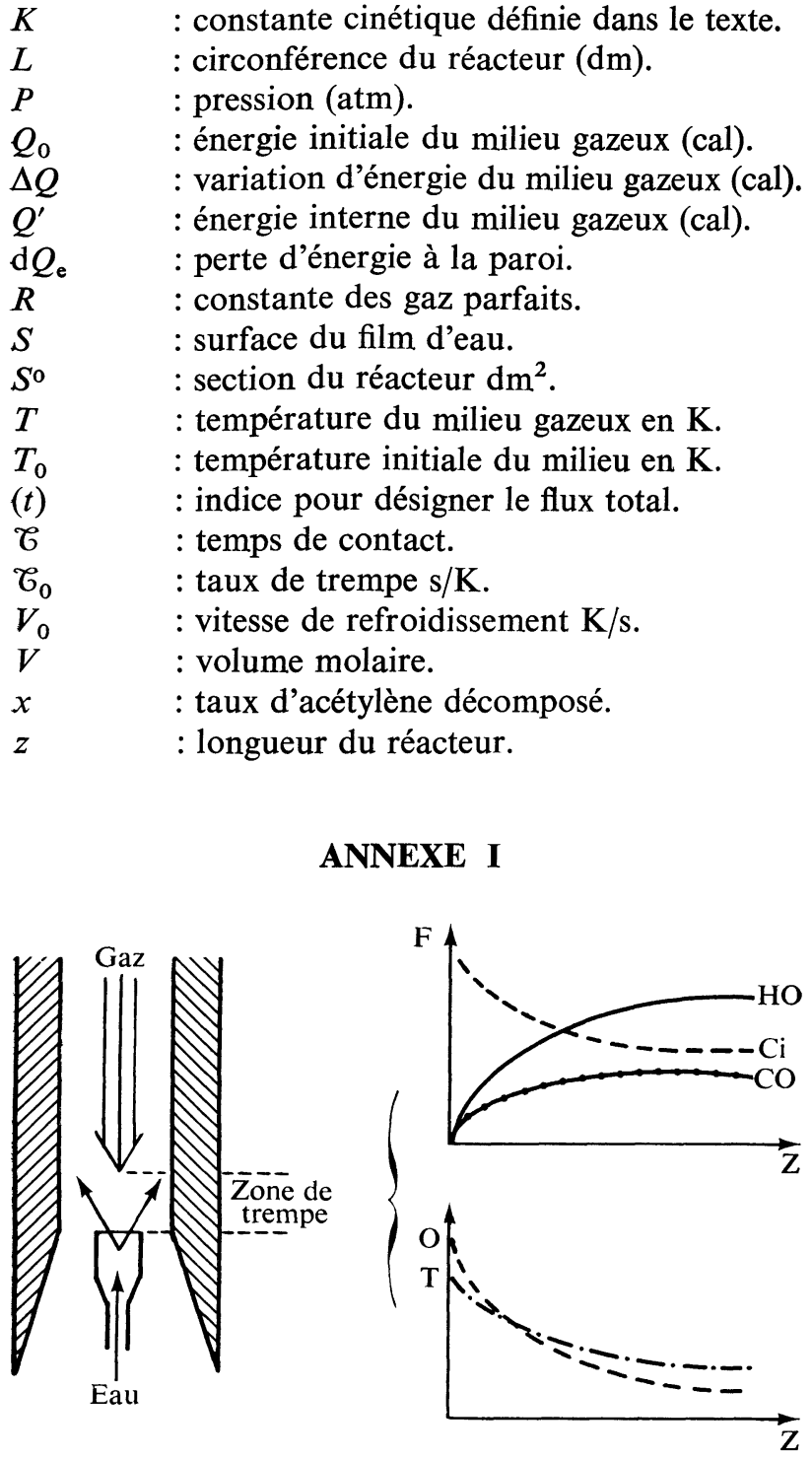

Schéma du réacteur de trempe.
On assiste d'une part à une vaporisation de l'eau selon la réaction :

$$
\mathrm{H}_{2} \mathrm{O} \text { liq } \rightarrow \mathrm{H}_{2} \mathrm{O} \text { vap }+\Delta H^{\prime} \text { (constante cinétique } k^{\prime} \text { ) }
$$

ce qui entraîne une baisse de la température du mélange, et d'autre part, la combustion de l'acétylène selon la réaction :

$$
\begin{aligned}
\mathrm{C}_{2} \mathrm{H}_{2}+2 \mathrm{H}_{2} \mathrm{O} \text { vap } \rightarrow & 2 \mathrm{CO}+3 \mathrm{H}_{2}+ \\
& +\Delta H(\text { constante cinétique } k) .
\end{aligned}
$$

L'apparition de l'eau se représente par l'équation :

$$
\frac{1}{S_{\mathrm{r}}} \frac{\mathrm{d} F_{\mathrm{e}}}{\mathrm{d} z}=K^{\prime} S \frac{Q^{\prime}}{\Delta H^{\prime}+\left(T-T_{0}\right) C_{\mathrm{e}}}
$$

équation cinétique de décomposition de l'acétylène.

$$
\text { Soit }
$$

$$
\frac{\mathrm{d} x}{\mathrm{~d} z}=K S_{\mathrm{r}} \frac{(1-x)\left(F_{\mathrm{e}}-2 F_{\AA} X\right)}{F} \text { avec } \quad K=k \frac{P}{R T}
$$

bilan matière des produits gazeux.

Le débit gazeux correspond au gaz neutre $F_{\mathrm{i}}$, à l'ensemble des produits de décomposition de l'acétylène et à l'eau vaporisée. Il s'écrit :

$$
F_{1}=F_{1}+F_{\mathrm{e}}+F_{\mathrm{a}}^{0}(1+2 X)
$$

bilan thermique

$$
\begin{gathered}
Q^{\prime}=F_{\mathrm{j}} C_{\mathrm{j}} T=Q_{0}+\Delta Q \\
T=\frac{Q_{0}+\Delta Q}{\sum F_{\mathrm{j}} C_{\mathrm{j}}} \\
\frac{\mathrm{d} Q}{\mathrm{~d} z}=\Delta H F_{\AA} \frac{\mathrm{d} x}{\mathrm{~d} z}-\frac{\mathrm{d} F_{\mathrm{e}}}{\mathrm{d} z}\left(\Delta H^{\prime}+\left(T-T_{0}\right) C_{\mathrm{e}}\right)- \\
-L C_{\mathrm{g}}\left(T-T_{0}\right) .
\end{gathered}
$$

\section{Bibliographie}

[1] Baddour, F., Timmins, R. S., The application of plasma chemical processing (Pergamon Press) 1967.

[2] BolL, A. T., In Techniques and applications of Plasma chemistry (Wiley) 1974.

[3] Blaustein, B. D., Chemical Reaction in électrical discharges (American chemical Sociéty) 1969.

[4] Chaudron, G., Trombe, F., Traité des Hautes Températures (Masson) 1973.

[5] Delcroix, J. L., Matos Ferreira, C., Ricard, A., Atomes et Molécules Métastables dans les gaz ionisés (C. N. R.S.) 1975.

[6] International Round table on study and application of transport phenomena in thermal plasmas. Odeillo. Sept. 1975, (éd. C. Bonet. Labo ultraréfractaires, 66120 Odeillo, France).

[7] RapoulaR (R.), Phénomènes électriques dans les gaz (Dunod) 1963.

[8] McTaggard, F. K., Plasma chemistry in electrical Discharges (Elsevier) 1967.

[9] Von Engel, A., Ionized Gases (Clarendon Press, Oxford) 1905.
[10] Amman, P. R., Timmins, R. S., AICHE J. 12 (1966) 956.

[11] Amouroux, J., Rapakoulias, D., Ann. Chimie 5 (1976) 26.

[12] Amouroux, J., Talbot, Ann. Chimie 4 (1969) 351.

[13] Collongues, R., Leprince Ringuet, F., Bull. Soc. Chim. V (1962) 523.

[14] Brooks, J. D., Hesp, W. R., Rigby, D., J. Appl. Chem. 17 (1967) 225.

[15] Amouroux, J., Talbot, J., Chim. Ind. Génie Chim. 105 (1972) 1201.

[16] Druyvestein, M. J., Penning, F. M., Rev. Mod. Phys. 12 (1940) 87.

[17] Fauchais, P., Thèse Doctorat ès-Sciences. Poitiers 28 mars 1968.

[18] Gilmore, F. R., J. Quant. Spect. Rad. Trans. 5 (1965) 369.

[19] Hamblym, S. M. L., Reuben, B. G., Adv. Inorg. Chem. 17 (1975) 89.

[19] Hamblym, S. M. L., Reuben, B. G., Adv. Inorg. Chem. 17 (1975) 89.

[20] Ibberson, V. I., High temperature-High Pressure 1 (1969) 243.

[21] Stokes, C. S., Adv. Chem. Série 80 (1969) 390. 
[22] Thornton, J. D., Adv. Chem. Série 80 (1969) 372.

[23] Vurzel, F. B., Polak, L. S., Ind. Eng. Chem. 62 (1970) 8.

[24] Fauchais, P., Bourdin, E., La Chimie des Plasmas et ses débouchés à court terme sur des synthèses inorganiques à caractère industriel (Congrès National des Plasmas de la Société Française de Physique) (Paris 6-10 déc. 1976).

[25] Dumas, J. L., Garnier, B., J. Chim. Phys. 9 (1975) 1045.

[26] Goldberger, W. M., OXLEY, J. H., AICHE J. 9 (1963) 778.
[27] Amouroux, J., Etude de la trempe d'un gaz réactif à haute température (Congrès International on study and applications of transport phenomene in thermal plasma) (Odeillo 12-16 sept. 1975).

[28] Amouroux, J., Talbot, J., Ann. Chim. 3 (1958) 219.

[29] Amouroux, J., Pineau, J. N., Rapakoulias, D., Ann. Chim. 1 (1976) 227.

[30] Schulze, R. A., Dupont de Nemours, Chem. Ind. 11 (1968) 1539. 